\title{
Mid-Infrared Spectral Diagnostics of Luminous Infrared Galaxies
}

\author{
A. Petric and the GOALS collaboration \\ Spitzer Science Center, California Institute of Technology, USA
}

\begin{abstract}
We present a statistical analysis of 248 luminous infrared galaxies (LIRGs) which comprise the Great Observatories All-sky LIRG Survey (GOALS) observed with the Infrared Spectrograph (IRS) on-board Spitzer in the rest-frame wavelength range between 5 and $38 \mu \mathrm{m}$. The GOALS sample enables a direct measurement of the relative contributions of star-formation and active galactic nuclei (AGN) to the total infrared (IR) emission from a large, statistically complete sample of LIRGs in the local Universe.

Several diagnostics effective at isolating the AGN contribution to the Mid-infrared (MIR) emission using [NeV], [OIV] and [NeII] gas emission lines, the $6.2 \mu \mathrm{m}$ PAH equivalent width (EQW) and the shape of the MIR continuum are compared. The $[\mathrm{NeV}]$ line which indicates the presence of an AGN is detected in $22 \%$ of all LIRGs. The $6.2 \mu \mathrm{m} \mathrm{PAH} \mathrm{EQW,}[\mathrm{NeV}] / \mathrm{L}_{\mathrm{IR}},[\mathrm{NeV}] /[\mathrm{NeII}]$ and [OIV]/[NeII] ratios, and the ratios of $6.2 \mu \mathrm{m}$ PAH flux to the integrated continuum flux between 5.3 and $5.8 \mu \mathrm{m}$ suggest values of around $10 \%$ for the fractional AGN contribution to the total IR luminosity of LIRGs. The median of these estimates suggests that for local LIRGs the fractional AGN contribution to the total IR luminosity is $\sim 12 \%$. AGN dominated LIRGs have higher global and nuclear IR luminosities, warmer MIR colors and are interacting more than starburst (SB) dominated LIRGs. However there are no obvious linear correlations between these properties, suggesting that none of these properties alone can determine the activity and evolution of an individual LIRG.

A study of the IRAC colors of LIRGs confirms that methods of finding AGN on the basis of their MIR colors are effective at choosing AGN but $50 \%$ to $40 \%$ of AGN dominated LIRGs are not selected as such with these methods.
\end{abstract}

\section{BACKGROUND}

The Infrared Astronomical Satellite (IRAS) provided the first unbiased survey of the sky at mid-infrared (MIR) and far-infrared (FIR) wavelengths, giving us a comprehensive census of the infrared (IR) emission properties of galaxies in the local Universe. These luminous infrared galaxies (LIRGs: $\mathrm{L}_{\mathrm{IR}[8-1000 \mu \mathrm{m}]} \geq 10^{11} \mathrm{~L}_{\odot}$, with $\mathrm{L}_{\mathrm{IR}}$ as defined in [1]) emit the bulk of their energy in the far-infrared. At the highest luminosities, ultraluminous infrared galaxies (ULIRGs: $\mathrm{L}_{\mathrm{IR}[8-1000 \mu \mathrm{m}]} \geq 10^{12} \mathrm{~L}_{\odot}$ ) have a space density that is 1.5-2 times higher than that of optically selected QSOs, the only other known objects with comparable bolometric luminosities [2]. LIRGs account for $\geq 50 \%$ of the total $24 \mu \mathrm{m}$ galaxy population with $f_{24 \mu \mathrm{m}} \geq 80 \mathrm{Jy}$ and for $\sim 50 \%$ of the comoving star formation density at $\mathrm{z} \sim 1$ [e.g. 3, 4, 5]. Unlike ULIRGs, LIRGs span the full range of galaxy interactions from non-merging spirals to late stage mergers, bridging the luminosity gap between SBs and QSOs. GOALS targets a complete sample of 202 systems in the local Universe selected from the IRAS Revised Bright Galaxy Sample [RBGS 6]. GOALS brings together HST (UV, optical and NIR), Spitzer (imaging and spectroscopy), GALEX, Chandra, and NIR ground-based spectroscopic data to understand 
low-z $(\leq 0.088)$ LIRGs. The broad wavelength coverage of this study can be used to trace the young and old stars, the dust, the hot ionized gas, and the warm molecular gas in LIRGs, making this data set unmatched in its power to shed light on the genesis of starbursts and the growth of black holes in interacting galaxies locally. An outline of the GOALS project and a multi-wavelength analysis of the LIRG VV340 are given in [7].

\section{RESULTS AND DISCUSSION}

The high resolution ( $\mathrm{SH}$ and $\mathrm{LH}$ ) LIRG spectra are dominated by atomic fine-structure lines of $\mathrm{Ne}, \mathrm{O}, \mathrm{Si}, \mathrm{S}$ as well as warm $\mathrm{H}_{2}$. The application of several methods to determine the relative contribution of star formation versus AGN to the IR luminosity of LIRGs from MIR spectra are presented. All wavelengths discussed here are rest-frame values.

The [NeV] $14.322 \mu \mathrm{m}$ and [OIV] $25.890 \mu \mathrm{m}$ lines are detected in $22 \%$ and $53 \%$ of all LIRG nuclei respectively while the $6.2 \mu \mathrm{m}$ PAH and [NeII] $12.8 \mu \mathrm{m}$ features are detected in $98 \%$ and $100 \%$ of the sample. Diagnostics using the $[\mathrm{NeV}] /[\mathrm{NeII}]$, [OIV]/[NeII] line flux ratios, the $6.2 \mu \mathrm{m}$ PAH EQW and the MIR continuum shape suggest that in $10 \%$ of local LIRGs the AGN dominates the bolometric luminosity (Figures 1,2 and 3). The vast majority of LIRGs are SB dominated. The fraction of local LIRG IR emission coming from an AGN as estimated in the mid infrared is approximately two times larger than that seen in normal galaxies $(\sim 5 \%)$ and, about three to four times lower than that seen in ULIRGs ( $\sim 30-40 \%)$.

Summing the bolometric luminosity contributed by each AGN in the sample and dividing by the total IR luminosity of all the LIRGs suggests that AGN are responsible for $\sim 12 \%$ of the total bolometric luminosity of local LIRGs.

In LIRGs there are no strong correlations between the fraction of IR luminosity from an AGN and the total or nuclear $24 \mu \mathrm{m}$ luminosity, 24 to $60 \mu \mathrm{m}$ flux ratios or the interaction stage of the system. However AGN dominated LIRGs tend to be brighter at $24 \mu \mathrm{m}$ and to have warmer IR colors than starburst dominated LIRGs.

By separating the GOALS sources according to merger stage it is found that there is a significant increase in the fraction of AGN dominated sources among those galaxies in the latest stages of interaction. This trend is driven by the ULIRGs in the sample, since these objects tend to be late stage mergers and have larger AGN fractions than the LIRGs. This is consistent with findings of previous investigations using optical diagnostics for LIRGs, MIR studies of ULIRGs and PG QSOs and with models which predict that mergers of gas-rich spirals fuel both star-formation and accretion onto a super-massive black hole.

An investigation of the IRAC colors (i.e. [3.6 $\mu \mathrm{m}]-[4.5 \mu \mathrm{m}]$ flux versus [5.8 $\mu \mathrm{m}]-$ [8 $\mu \mathrm{m}$ ] flux), as introduced in [8], of LIRGs indicates that only $50 \%$ of objects with a significant AGN contribution to the MIR emission fall within the range typically associated with AGN. The [9] AGN IRAC color criteria select a slightly higher fraction of the AGN dominated LIRGs (64\%), at the expense of also including 11 LIRGs that appear SB dominated from their IRS spectra.

IR number counts have been used by several groups of researchers to determine the star-formation history of our universe. The measurements provided in this paper are important in this context because they provide an estimate of the fraction of local LIRG 
IR emission coming from an AGN and thus not from star-formation. If the physical properties of LIRGs do not strongly evolve between redshifts 0 and 1, then the statistics derived here can be used to ascertain the properties IR luminous systems at redshfits where their numbers as well as contribution to the total energy production in the universe is substantial.

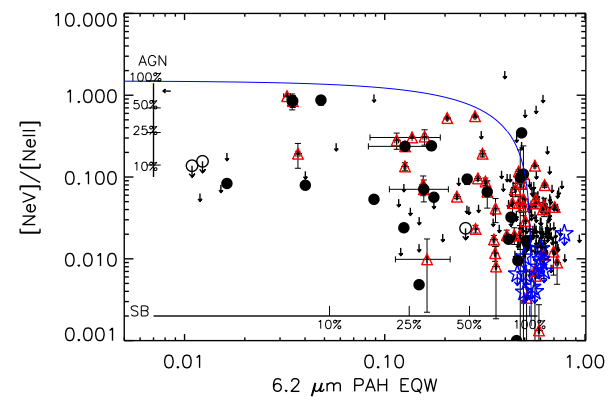

FIGURE 1. Mid-infrared $[\mathrm{NeV}] /[\mathrm{NeII}]$ versus $6.2 \mu \mathrm{m}$ PAH EQW excitation diagram. The triangles are $[\mathrm{NeV}]$ detections while the arrows are upper limits. In all cases $1 \sigma$ error bars are shown. The circles indicate ULIRGs where the filled symbols show detections and empty symbols upper limits. The empty stars mark upper limits for star-burst galaxies from [10]. The solid vertical and horizontal lines indicate the fractional AGN and starburst contribution to the MIR luminosity from the $[\mathrm{NeV}] /[\mathrm{NeII}]$ (vertical) and $6.2 \mu \mathrm{m}$ PAH EQW (horizontal) assuming a simple linear mixing model. In each case, the $100 \%, 50 \%$, $25 \%$, and $10 \%$ levels are marked. The $100 \%$ level is set by the average detected values for the $[\mathrm{NeV}] / \mathrm{NeII}]$ and $6.2 \mu \mathrm{m}$ EQW among AGN and starbursts respectively, as discussed in [11]. The curved line traces where the summed SB and AGN contribution equals $100 \%$.

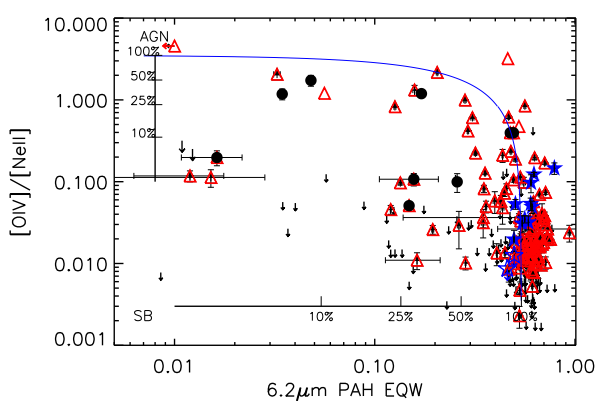

FIGURE 2. Mid-infrared [OIV]/[NeII] versus $6.2 \mu \mathrm{m}$ PAH EQW excitation diagram. Symbols have the same definition as in Figure 1 except that triangles indicate [OIV] detections. 


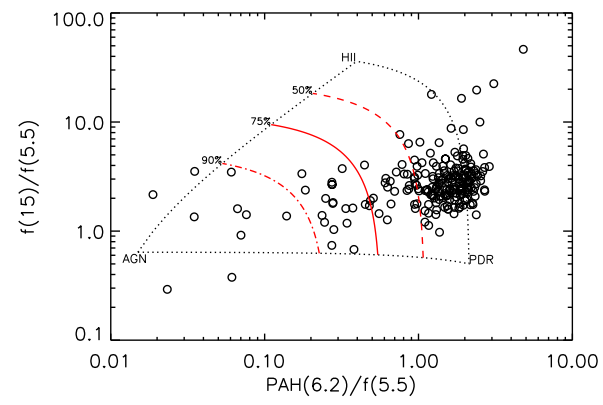

FIGURE 3. Mid-infrared diagnostic diagram comparing the integrated continuum flux from $14-15 \mu \mathrm{m}$, the integrated continuum flux from 5.3-5.5 and the 6.2 $\mu \mathrm{m}$ PAH flux. The three vertices, labeled as AGN, HII and PDR, represent the positions of 3C273 from [12], M17 and NGC 7023 from [13]. These vertices were chosen to facilitate comparison with ULIRGs as presented in [11] and [14]. The lines from left to right indicate a $90 \%, 75 \%$ and $50 \%$ fractional AGN contribution to the nuclear MIR luminosity respectively.

\section{REFERENCES}

1. Sanders, D. B., Mirabel, I. F., ARAA 34, 749 (1996)

2. Schmidt, M., Green, R. F., ApJ 269, 352 (1983)

3. Le Floc'h, E., Papovich, C., Dole, H., et al., ApJ 632, 169 (2005)

4. Caputi, K. I., Dole, H., Lagache, G., et al., A\&A 454, 143 (2006)

5. Magnelli, B., Elbaz, D., Chary, R. R., et al., A\&A 496, 57 (2009)

6. Sanders, D. B., Mazzarella, J. M., Kim, D., Surace, J. A., Soifer, B. T., AJ 126, 1607 (2003)

7. Armus, L., Mazzarella, J. M., Evans, A. S., et al., PASP 121, 559 (2009)

8. Stern, D., Eisenhardt, P., Gorjian, V., et al., ApJ 631, 163 (2005)

9. Lacy, M., Storrie-Lombardi, L. J., Sajina, A., et al., ApJS 154, 166 (2004)

10. Bernard-Salas, J., Spoon, H. W. W., Charmandaris, V., et al., ApJS 184, 230 (2009)

11. Armus, L., Charmandaris, V., Bernard-Salas, J., et al., ApJ 656, 148 (2007)

12. Weedman, D. W., Hao, L., Higdon, S. J. U., et al., ApJ 633, 706 (2005)

13. Peeters, E., Spoon, H. W. W., Tielens, A. G. G. M., ApJ 613, 986 (2004)

14. Brandl, B. R., Bernard-Salas, J., Spoon, H. W. W., et al., ApJ 653, 1129 (2006) 